\title{
UMA ANÁLISE SOBRE AS CHUVAS NO CEARÁ BASEADA NOS EVENTOS DE EL NIÑO, LA NIÑA E NO DIPOLO DO SERVAIN DURANTE A ESTAÇÃO CHUVOSA
}

\author{
RODRIGUES, Bruno Dias - bruno.rodrigues@funceme.br \\ Fundação Cearense de Meteorologia e Recursos Hídricos / FUNCEME \\ COUTINHO, Maytê Duarte Leal - mayte.coutinho@inmet.gov.br \\ Instituto Nacional de Meteorologia / INMET \\ SAKAMOTO, Meiry Sayuri - meiry@funceme.br \\ Fundação Cearense de Meteorologia e Recursos Hídricos / FUNCEME \\ JACINTO, Leandro Valente - leandro.jacinto@funceme.br \\ Fundação Cearense de Meteorologia e Recursos Hídricos / FUNCEME
}

Submetido em: 01/09/2020

Aceito para publicação em: 23/04/2021

Publicado em: 10/05/2021

DOI: http://dx.doi.org/10.5380/abclima.v28i0.76238

\begin{abstract}
RESUMO: Ao longo dos anos, estudos têm abordado a relação dos oceanos Pacífico (OP) e Atlântico (OA) com as chuvas no Nordeste brasileiro (NEB). No entanto, nessa região, o regime pluviométrico apresenta períodos e volumes distintos, sendo importante realizar uma análise específica para diferentes áreas, visando entender as eventuais particularidades. Nesta pesquisa, o objetivo principal foi identificar como as chuvas se comportaram diante de eventos de El Niño (EN) e La Niña (LN) e diante da condição do Dipolo de Servain (DS) no AO nos trimestres Fevereiro-Março-Abril (FMA) e Março-AbrilMaio (MAM) durante a estação chuvosa no Ceará. Adicionado a isso, investigaram-se os meses Fevereiro-Março-Abril-Maio (FMAM) separadamente como forma de melhor compreender a variabilidade existente dentro da estação chuvosa. Os resultados indicaram que o fenômeno EN não acarretou na diminuição das chuvas em dois anos, 1995 e 2019, pois o fenômeno apresentou intensidade fraca nos trimestres FMA e MAM da estação chuvosa e a presença de um DS negativo no OA nesse período. Com relação aos anos de $L N$, verificou-se que a combinação de $L N$ de intensidade fraca e moderada e DS negativo induzem a chuvas acima da média na estação chuvosa. Enquanto, a presença de LN de intensidade fraca e DS neutro ou positivo refletem em chuvas na média ou abaixo da média.
\end{abstract}

PALAVRAS-ChAVE: El Niño; La Niña; Dipolo do Servain; Estação Chuvosa; Ceará.

AN ANALYSIS OF RAIN IN CEARÁ BASED ON THE EVENTS OF EL NIÑO, LA NIÑA AND THE DIPOLE OF SERVAIN DURING THE RAINY STATION

ABSTRACT: Over the years, studies have addressed the relationship between the Pacific (PO) and Atlantic (AO) oceans with rainfall in Northeast Brazil (NEB). However, in this region, the rainfall regime has different periods and volumes, and it is important to carry out a specific analysis for different areas, in order to understand any particularities. In this research, the main objective was to identify how the rains behaved in the face of the events of El Niño (EN) and La Niña (LN) and the condition of the Servain Dipole (SD) in the AO, established in the February-March-April quarters (FMA) and March-April-May (MAM) during the rainy season in Ceará. Added to this, the months February-March-AprilMay (FMAM) were investigated separately as a way to better understand the variability existing within the rainy season. The results indicated that the EN phenomenon did not cause a decrease in rainfall in two years, 1995 and 2019, as the phenomenon had a weak intensity in the FMA and MAM quarters of the rainy season and the presence of a 
negative DS in the OA in that period. Regarding the years of LN, it was found that the combination of LN of weak and moderate intensity and negative DS induces aboveaverage rainfall in the rainy season. Meanwhile, the presence of weak intensity LN and neutral or positive DS reflect in average or below average rainfall.

KEYWORDS: El Niño; La Niña; Servain Dipole; Rainfall Season; Ceará.

ANÁLISIS DE LLUVIA EN CEARÁ BASADO EN LOS EVENTOS DE EL NIÑO, LA NIÑA Y EL DIPOLE DE SERVAIN DURANTE LA ESTACIÓN DE LLUVIAS

RESUMEN: A lo largo de los años, los estudios han abordado la relación entre los océanos Pacífico (OP) y Atlántico (OA) con las precipitaciones en el noreste de Brasil (NEB). Sin embargo, en esta región, el régimen de lluvias tiene diferentes períodos y volúmenes, por lo que es importante realizar un análisis específico para diferentes áreas, con el fin de comprender las particularidades. En esta investigación, el objetivo principal fue identificar cómo se comportaron las lluvias ante los eventos de El Niño (EN) y La Niña (LN) y en vista de la condición del Dipolo Servain (DS) en el AO, estableció en los trimestres febrero-marzo-abril (FMA) y marzo-abril-mayo (MAM) durante la época de lluvias en Ceará. Sumado a esto, los meses febrero-marzo-abril-mayo (FMAM) se investigaron por separado como una forma de comprender mejor la variabilidad existente dentro de la temporada de lluvias. Los resultados indicaron que el fenómeno EN no resultó en una disminución de las precipitaciones en dos años, 1995 y 2019, ya que el fenómeno tuvo una intensidad débil en los trimestres FMA y MAM de la temporada de lluvias y la presencia de un DS negativo en el OA. en ese período. En cuanto a los años de $L N$, se encontró que la combinación de LN de intensidad débil y moderada y DS negativo induce precipitaciones superiores al promedio en la época de lluvias. Mientras tanto, la presencia de LN de intensidad débil y DS neutra o positiva se refleja en la precipitación media o por debajo de la media.

PALABRAS CLAVE: El Niño, La Niña, Dipolo do Servain, Temporada de lluvias, Ceará.

\section{INTRODUÇÃO}

O estado do Ceará está localizado na região Norte do Nordeste Brasileiro (NNEB), e conforme Marengo et al. (2010) tem o seu período chuvoso nos meses de fevereiro a maio, com volumes pluviométricos médios em torno dos 600mm. De acordo com Alves e Repelli (1992) e Andreoli et al. (2004), a Temperatura da Superfície do Mar (TSM) dos OP e OA é conhecida como um dos fatores que interfere diretamente na qualidade da estação chuvosa. Alves (1999) considera que as análises mensais da TSM dos OP e OA, no período que antecede a estação chuvosa e na estação chuvosa do NNEB possuem influência na variabilidade da precipitação da região.

Por conceito, o aquecimento (resfriamento) anômalo das águas superficiais no OP tropical por períodos prolongados indica uma condição de EN (LN). E consequentemente, essas relações estão associadas a uma tendência de chuvas abaixo (acima) da média climatológica no NEB, conforme descrito por Alves (2002). Enquanto, no OA tropical, conforme mostrado por Servain (1991) e Nobre et al. (1996), tem-se a presença de um gradiente de TSM, em que, águas mais aquecidas no norte do que no sul da bacia (Dipolo Positivo - DP) e águas mais aquecidas no sul do que no norte da bacia (Dipolo Negativo - DN) podem ser associadas a chuvas abaixo (acima) da média climatológica no NEB.

Essas relações citadas acima, que já são conhecidas cientificamente, refletem em anos secos e chuvosos, como observado historicamente e recentemente na região nos anos de 2012 a 2016, com anos secos e 2004 e 2009, com anos chuvosos. Aliado a isso, trazem consequências para alguns setores como a agricultura e os recursos hídricos. Já que, essas áreas 
necessitam de informações antecipadas para suas tomadas de decisão e qualquer condição de escassez ou excesso de chuvas pode causar situações delicadas no seu gerenciamento.

O aperfeiçoamento da previsão climática e o fato do NEB ser uma das regiões que apresenta um grau de habilidade considerável para representar o comportamento da precipitação sazonal, vem servindo de referência para o planejamento local e obtendo resultados satisfatórios, segundo Martins e Vasconcelos (2017). No entanto, incertezas podem ocorrer pelo fato do sistema climático terrestre ser caótico, sendo necessário análises baseadas em dados observados passados, a partir de eventos similares que ocorreram em períodos distintos em um (a) determinado (a) local/região.

Ao mesmo tempo, a ideia geral que se tem quando se aborda, principalmente a questão de EN e LN, é que esses fenômenos trarão de qualquer maneira impactos na redução ou aumento das chuvas no NEB. Rodrigues et al. (2011) e Reboita e Santos (2015) investigaram essa questão e encontraram características no OP e no OA que podem ou não potencializar a falta de chuva, e/ou, até mesmo, não influenciar no regime de chuva do NEB. Assim, variações na TSM do OP as quais desrespeitam a intensidade e a evolução desses fenômenos dentro dos trimestres FMA e MAM fornecem algumas respostas que podem, não necessariamente, obedecer o que é estabelecido pela literatura científica. Já com relação ao OA, tem-se estudos que comprovam a sua importância para com as chuvas da região. No entanto, poucas análises foram exploradas sobre a questão da intensidade e evolução das anomalias de TSM dentro da estação chuvosa.

Logo, compreender se a relação dos oceanos com a estação chuvosa no Ceará interfere em todas as situações em que atuam, torna-se fundamental. Por tal razão, neste trabalho, o objetivo principal é identificar como as chuvas se comportam diante de eventos de EN (LN) e diante da condição do DS nos trimestres FMA e MAM durante a estação chuvosa no Ceará (FMAM). Além disso, discute-se sobre a variação mensal dos desvios percentuais de precipitação dentro da estação chuvosa no Estado. Cabe salientar que a pequena quantidade de casos escolhidos se deu em virtude dos dados do DS estarem disponíveis desde 1964, sendo de fato esta análise utilizada de uma maneira complementar ao tema em discussão.

\section{MATERIAL E MÉTODOS}

\section{ÁREA DE ESTUDO}

O Ceará está localizado no NNEB e pertence ao semiárido nordestino (Figura 1), apresentando precipitação média em torno dos $600 \mathrm{~mm}$ na sua estação chuvosa, que é de fevereiro à maio. As médias históricas mensais de fevereiro à maio são respectivamente: $118,6 \mathrm{~mm}, 203,4 \mathrm{~mm}, 188 \mathrm{~mm}$ e $90,6 \mathrm{~mm}$. De acordo com Ferreira e Da Silva Mello (2005), nesse período, o principal sistema meteorológico atuante e responsável pelas chuvas, é a Zona de Convergência Intertropical (ZCIT). No entanto, a região pode ter influência de outros sistemas como os Vórtices Ciclônicos de Altos Níveis (VCAN), os Sistemas Frontais que se aproximam do sul do NEB e os sistemas locais, como as brisas marítima e terrestre. 


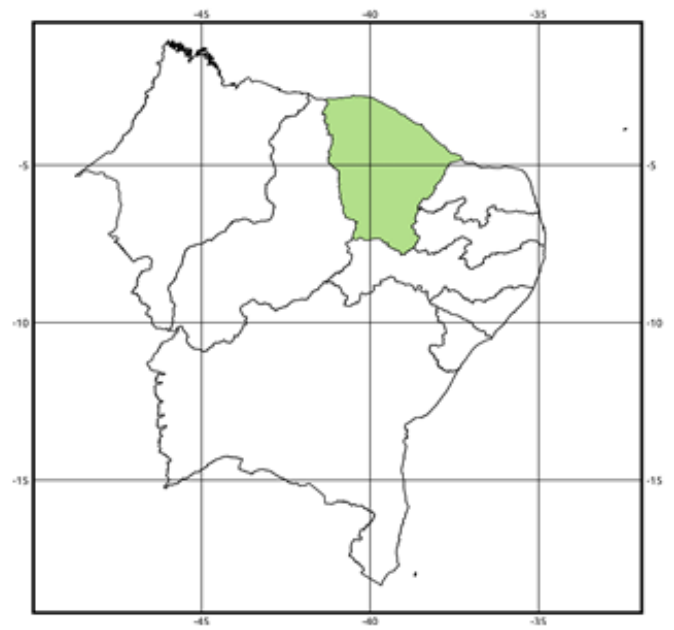

Figura 1 - Localização do Nordeste Brasileiro e em destaque o Estado do Ceará.

\section{PRECIPITAÇÃO}

Utilizaram-se dados de precipitação, disponibilizados pela Fundação Cearense de Meteorologia e Recursos Hídricos (FUNCEME) para o Ceará de 1974 a 2019 nos meses de fevereiro a maio. Essas informações são coletadas diariamente e têm por base aproximadamente 550 pluviômetros convencionais (Figura 2) distribuídos por todo o estado, sendo que o valor médio é obtido pelo método de Thiessen, no qual se estabelece pesos para cada posto pluviométrico na hora do cálculo estatístico médio. Essas informações estão disponíveis no site da FUNCEME (www.funceme.br) por meio do produto "Calendário de Chuvas".



Figura 2 - Localização dos pluviômetros convencionais da FUNCEME no Estado do Ceará.

\section{INDÍCE OCEÂNICO (ONI)}

Para a identificação dos ENs e LNs, o Índice Oceânico (ONI) referente ao Pacífico, do Climate Prediction Center/National Oceanic and Atmospheric Administration (CPC/NOAA) foi utilizado (Figura 3), pois considera as anomalias de TSM na região 3.4 do Oceano Pacífico central (entre as latitudes de $5^{\circ} \mathrm{N}$ e $5^{\circ} \mathrm{S}$ e as longitudes de $120^{\circ} \mathrm{W}$ e $180^{\circ} \mathrm{W}$ ), caracterizando o fenômeno a partir de anomalias positivas ou negativas acima de $0,5^{\circ} \mathrm{C}$ persistentes por mais de cinco 
trimestres consecutivos. As anomalias de TSM que apresentam valores positivos ou negativos entre $0,5^{\circ} \mathrm{C}$ e $1,0^{\circ} \mathrm{C}$ representam uma intensidade fraca, enquanto entre $1,0^{\circ} \mathrm{C}$ e $1,5^{\circ} \mathrm{C}$, correspondem a intensidade média e, acima de $1,5^{\circ} \mathrm{C}$, a intensidade forte.

\section{DÍPOLO DO SERVAIN (DS)}

De acordo com Servain (1991), o DS, referente ao Atlântico, leva em conta as condições de TSM nas latitudes de $28^{\circ} \mathrm{N}$ a $20^{\circ} \mathrm{S}$ e nas longitudes de $60^{\circ} \mathrm{W}$ a $15^{\circ} \mathrm{E}$, no qual a bacia norte se localiza entre $5^{\circ} \mathrm{N}$ e $28^{\circ} \mathrm{N}$ e a bacia sul entre $5^{\circ} \mathrm{N}$ e $20^{\circ} \mathrm{S}$ (Figura 3). A metodologia para a obtenção dos valores de TSM, consiste em calcular as médias mensais de TSM nas duas bacias norte e sul do OA tropical. Com a climatologia mensal e os dados de TSM mensal, obtém-se o desvio padrão da TSM em cada bacia. Com isso, a diferença entre a bacia norte e a bacia sul identificam o padrão de DS positivo (águas mais quentes na bacia norte que na bacia sul do OA) e DS negativo (águas mais quentes na bacia sul que na bacia norte do OA). Maiores detalhes podem ser consultados no site da FUNCEME.

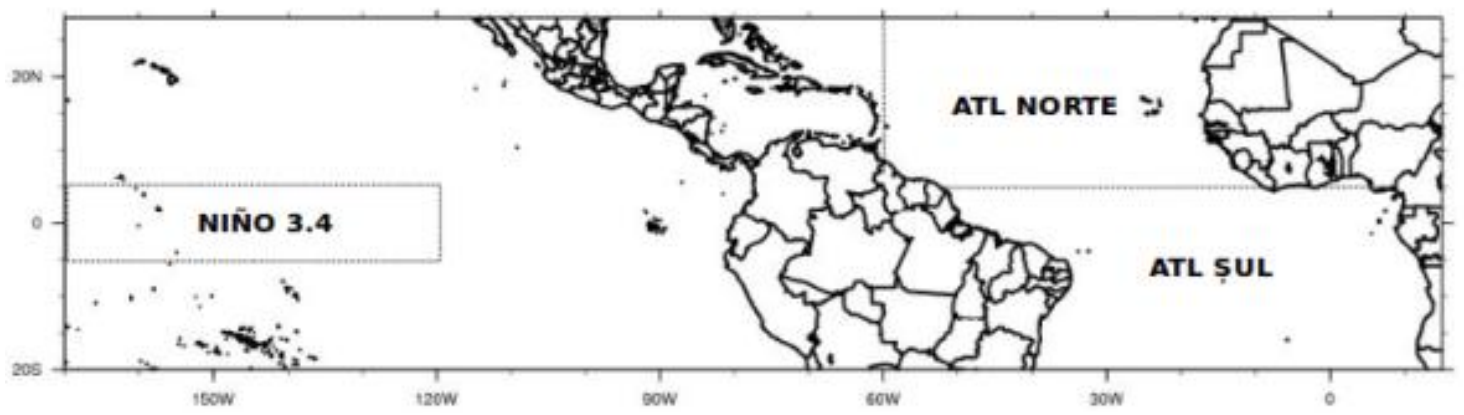

Figura 3 - Representação da localização correspondente ao Niño 3.4 no OP e as áreas utilizadas para o cálculo do DS no OA norte e sul.

\section{RESULTADOS E DISCUSSÕES \\ ÍNDICE OCEÂNICO E DIPOLO DO SERVAIN EM ANOS DE EN}

A Figura 4 relaciona os valores de anomalia de TSM do índice ONI e os valores das anomalias trimestrais média do DS com a precipitação observada nos meses de fevereiro à maio no estado do Ceará.

No total, desde 1974, observaram-se nove casos de EN no OP presentes em FMA e em FMA e MAM, sendo quatro deles acompanhados de um DS positivo, três de um DS negativo e dois neutros no OA, não mantendo, assim, uma configuração padrão. Destes nove, apenas em dois anos, 1995 e 2010, não houve persistência do EN no trimestre MAM, já que o EN passou a condição de neutralidade. E com relação ao DS, pode-se notar um contraste, com DS negativo no primeiro e DS positivo, no segundo.

De forma geral e com base na Figura 4, percebe-se que os anos com chuvas abaixo da média histórica na estação chuvosa no Ceará se deram pela associação de EN persistente em FMA e MAM, de intensidade moderada ou forte no OP e DS positivo no OA, como em 1983, 1987 e 1992 e por EN de 
intensidade moderada e forte e DS neutro, como nos anos de 1998 e 2016 . Em contrapartida, os anos de precipitações dentro da média e acima da média ocorreram quando se tinha um EN de intensidade fraca ou neutralidade no OP e um DS negativo no OA, como em 1995 e 2019. O ano de 2015, apresentou condições oceânicas similares aos dois últimos, porém, as chuvas ficaram abaixo da média histórica. Nesse ano, mesmo com um padrão semelhante a 2019, há um aumento nos valores de anomalia de TSM do OP em MAM, diferentemente de todos os outros anos analisados, enquanto no OA, nesse mesmo trimestre, nota-se uma redução dos valores de anomalia de TSM, embora permaneça com um DS negativo.

Resultados semelhantes foram encontrados nos estudos de Reboita e Santos (2015), que identificaram que na presença de EN e do OA com anomalias mais quentes na parte norte do que na parte mais ao sul, as condições de seca se intensificam no NNEB, como observado nos anos de 1983 e 1992. Ainda, segundo, Martins et al. (2017) e Rodrigues et al. (2011), esses padrões oceânicos favorecem o posicionamento da ZCIT mais ao norte da sua posição climatológica. Com respeito ao ano de 2010, Martins e Vasconcelos (2017), retrataram que em termos de observação dos valores de precipitação, a parte NNEB sofreu com a falta de chuvas e, em oposição, a parte mais sul do NEB recebeu volumes de chuva satisfatórios, dentro da média e acima da média.

Já, nos casos de 1995, 2015 e 2019, observa-se um EN de intensidade fraca combinado com um DS negativo, com chuvas acima e dentro da média nos anos de 1995 e 2019, e de chuvas abaixo da média, em 2015. Rodrigues et al. (2011), destacaram em suas análises que os anos com EN de curta duração e fraca intensidade estão relacionados a eventos úmidos no NEB, ocorrendo neste caso precipitações dentro ou acima da média, como verificado nos casos de 1995 e 2019. Mas em relação ao caso de 2015, isso não aconteceu, pois, o EN estava em processo de evolução das anomalias de TSM. Do ponto de vista dinâmico, nesse evento de EN considerado como muito forte, em 2015-2016, Medeiros (2019), identificou que o déficit de chuva esteve associado a um ramo descendente na circulação de Walker e a movimentos subsidentes ligados a ZCIT.

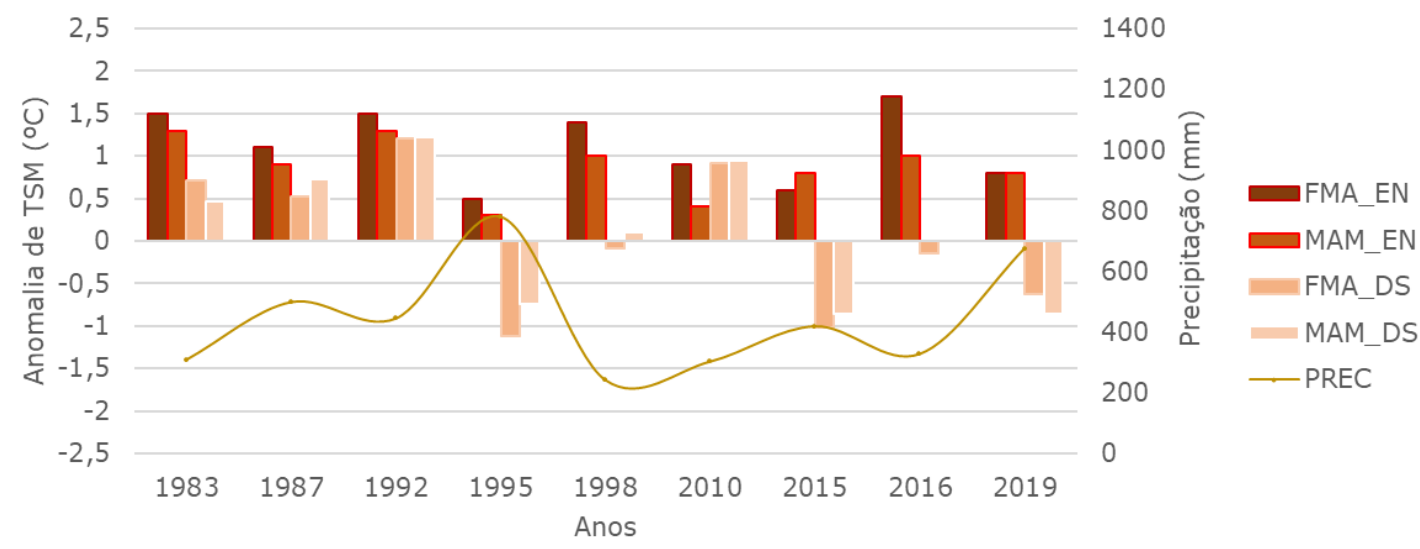

Figura 4 - Índice ONI referente ao EN nos trimestres FMA e MAM (barras em vermelho) e o DS em FMA e MAM (barras em laranja) e a Precipitação média em FMAM em anos de EN no Ceará (linha). Fonte: FUNCEME/NOAA. 


\section{DESVIOS PERCENTUAIS DE PRECIPITAÇÃO MENSAL EM ANOS DE EN}

A distribuição das chuvas nos meses que compõem a estação chuvosa, FMAM, pode ser analisada através dos valores dos desvios percentuais em anos de EN (Figura 5). Cabe salientar, que os quatro meses possuem valores médios mensais diferentes $e$, consequentemente, as variações apresentam um peso diferente para cada mês.

No mês de fevereiro (Figura 5), observou-se que em quatro casos, as chuvas se comportaram dentro da normal e acima da normal histórica, nos anos de 1983, 1992, 1995 e 2019. Nos três últimos anos citados, esses valores representam percentuais de 25 a $28 \%$ da média de chuva observada para o quadrimestre FMAM no Ceará. Como fevereiro está ligado ao início da estação, essas chuvas auxiliam na etapa de crescimento das culturas e colaboram com o processo de recarga dos açudes que passam praticamente o segundo semestre do no anterior com as águas das chuvas da estação passada, a não ser que o mês de janeiro, na pré-estação chuvosa, receba volumes acima do esperado, como referido por Alves et al. (2004) em 2004 e também recentemente em 2016. Nos anos de 1998, 2010 e 2016, esse mês apresentou desvios negativos que variam entre $55 \%$ e $80 \%$. Essa situação de estiagem, quase sempre é responsável por problemas de perdas de lavoura. No ano de 2015, mesmo com a situação de EN fraco, as chuvas ficaram $20 \%$ abaixo da média para o mês. A configuração do DS negativo se mostrou um fator que mesmo na presença de EN, contribuiu para que não se tivesse uma escassez de chuva tão acentuada como em 2010 e 2016.

Para o mês de março (Figura 5), apenas em um único ano ocorreu um valor acima do esperado, em 1987. Enquanto em 1995 e 2019, as chuvas ficaram dentro do esperado para o mês e, em 2015, levemente abaixo da normal histórica. Nos três últimos anos citados, nota-se a importância e persistência do OA com DS negativo, principalmente sobre o trimestre FMA, como mencionado anteriormente. Embora o mês de março de 1987 tenha ficado com valores percentuais acima da média, Marengo et al. (2011), atribuiu ao EN, as fortes perdas na produção de grãos do Ceará, as quais chegaram a $75 \%$. Nesse mês, o ano de 2010, foi o que teve maior desvio negativo de aproximadamente $50 \%$. Por ser o mês que apresenta maior média de chuva, o que em termos percentuais representa 34\% das chuvas da estação, quando há desvios negativos, é necessário que haja boas chuvas nos outros principais meses, abril e fevereiro.

Por fim, nos meses de abril e maio (Figura 5), mostraram-se valores abaixo da média com exceção do ano de 1995 e 2019. No caso de 1995, com o EN passando a neutralidade no trimestre MAM e com o DS negativo, as chuvas se mostraram com desvios positivos tanto em abril quanto em maio, favorecendo para que a estação chuvosa ficasse com um valor médio de chuva por volta de $800 \mathrm{~mm}$. Entretanto, em 2019, os volumes médios de chuva ficaram dentro da média histórica, mostrando que mesmo com um EN de intensidade fraca, é possível que se tenha valores dentro da normalidade. Nos demais anos, os quais foram observados desvios percentuais negativos nos meses finais da estação chuvosa, consegue-se verificar uma semelhança na tendência de redução das chuvas em anos de EN com intensidade moderada e forte e DS positivo ou neutro. Isso, de certa forma está ligado a questão do deslocamento 
da ZCIT mais ao norte devido ao OA norte estar mais aquecido do que o OA sul, como mencionado anteriormente.
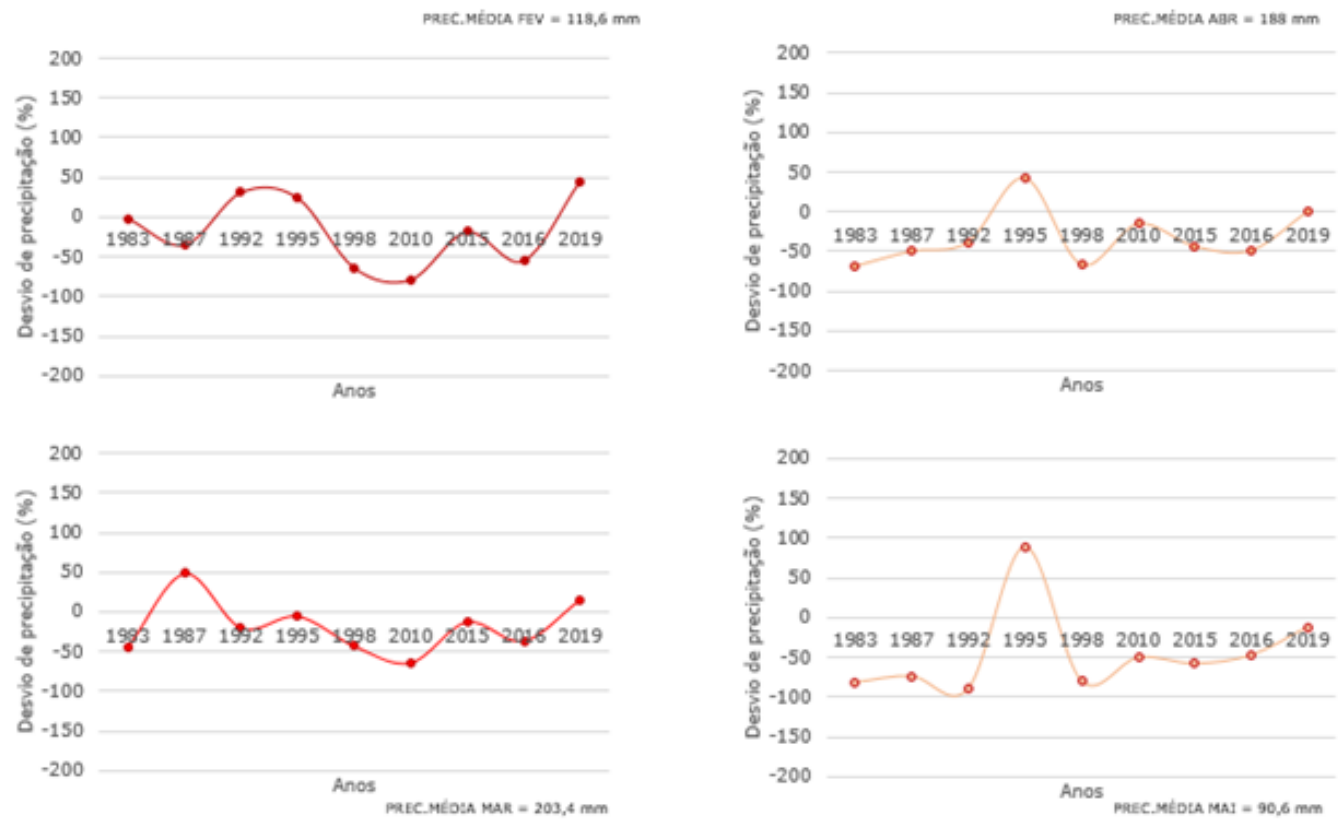

Figura 5 - Desvio percentual de precipitação mensal no Ceará em anos de EN. Fevereiro e março à esquerda nas cores em vermelho e abril e maio à direita nas cores em laranja. Fonte: FUNCEME.

\section{ÍNDICE OCEÂNICO E DIPOLO DO SERVAIN EM ANOS DE LN}

A Figura 6 relaciona os valores de anomalia de TSM do índice ONI e os valores das anomalias trimestrais média do DS com a precipitação observada nos meses de fevereiro a maio no estado do Ceará.

Dos 14 anos analisados, cinco anos, 1996, 2006, 2009, 2012 e 2018, não tiveram a continuidade da LN em MAM e, na maioria dos casos, o DS esteve com valores negativos no OA, com exceção de 1976, 2006, 2012 e 2018, em que o DS esteve neutro ou positivo (Figura 5).

De forma geral e com base na Figura 6, os maiores volumes de chuva no Ceará na estação chuvosa ocorrem com a combinação de LN fraco ou LN moderado com um DS negativo e com anomalias trimestrais em FMA e MAM que ultrapassam os valores de $1^{\circ} \mathrm{C}$. Já, os volumes de chuva que se encontram dentro da média ou abaixo da média histórica, apresentam uma relação com a presença de LN de intensidade fraca até o trimestre FMA e neutralidade em MAM e um DS neutro ou positivo.

Em grande parte dos casos em que houve o resfriamento das águas do $O P$, as anomalias dos trimestres FMA no OA se encontraram com valores similares ou mais baixos do que em MAM, evidenciando que houve um fortalecimento do DS no OA no trimestre MAM, como por exemplo no ano de 1974, um dos que tiveram as maiores médias de chuva no quadrimestre FMAM, 
alcançando os $1200 \mathrm{~mm}$. Nesse ano, o ONI apresentava um LN de intensidade moderada passando a fraca, de FMA para MAM. Alves e Souza (1997) constataram essa relação, mostrando que em anos de LN, os acumulados de chuva tendem a superar a média histórica no Ceará.

No entanto, Lucena et al. (2011) reforçaram o que foi observado por Moura e Shukla (1981), enfatizando que em episódios de LN, o OA tropical tem uma contribuição maior que o OP, já que a combinação de LN e um Gradiente Meridional do OA negativo, nesse caso equivalente a um DS negativo, trazem precipitações mais significativas e abundantes para o NEB. Essa combinação foi verificada em alguns episódios de $L N$ de intensidade fraca, persistentes em FMA e MAM e DS negativo, com anomalias acima de $-1^{\circ} \mathrm{C}$, nos anos de 1985,1989 e 2009, nos quais os volumes de chuva em FMAM ficaram entre 800 e $1200 \mathrm{~mm}$. Alves et al. (1997), identificaram um aumento de $15 \%$ a $20 \%$ da produção e da produtividade agrícola no Ceará em anos de LN. Da Silva Costa et al. (2015), mostraram a influência do LN na frequência de extremos de precipitação na estação chuvosa do NNEB, destacando 179 eventos no ano de 1985, indicando assim que há maior tendência de ocorrência de volumes de chuva extremos nos anos associados a LN.

Em contrapartida, quando essa configuração não ocorre e, se tem um LN de intensidade fraca e um DS neutro ou DS levemente positivo, as chuvas tendem a permanecer dentro da média, como em 1976, 2011 e 2018 ou até mesmo abaixo da média, como no ano de 2012 (Figura 5). Nesse último ano em especial, não apenas o NNEB sofreu com anomalias de precipitação negativa, mas grande parte do Brasil. Medeiros (2019), observou que entre os anos de 2012 e 2014, as chuvas no NNEB ficaram abaixo do esperado devido ao movimento descendente relacionado ao posicionamento da ZCIT, corroborando com os resultados encontrados por Marengo et al. (2016), os quais verificaram maior subsidência sobre o NEB. Martins e Magalhães (2016), retrataram a criticidade do abastecimento de água nos reservatórios cearenses, os impactos da falta de água no segundo semestre de 2012 e as quedas nas produções de milho e feijão, mandioca, frutas e hortaliças.

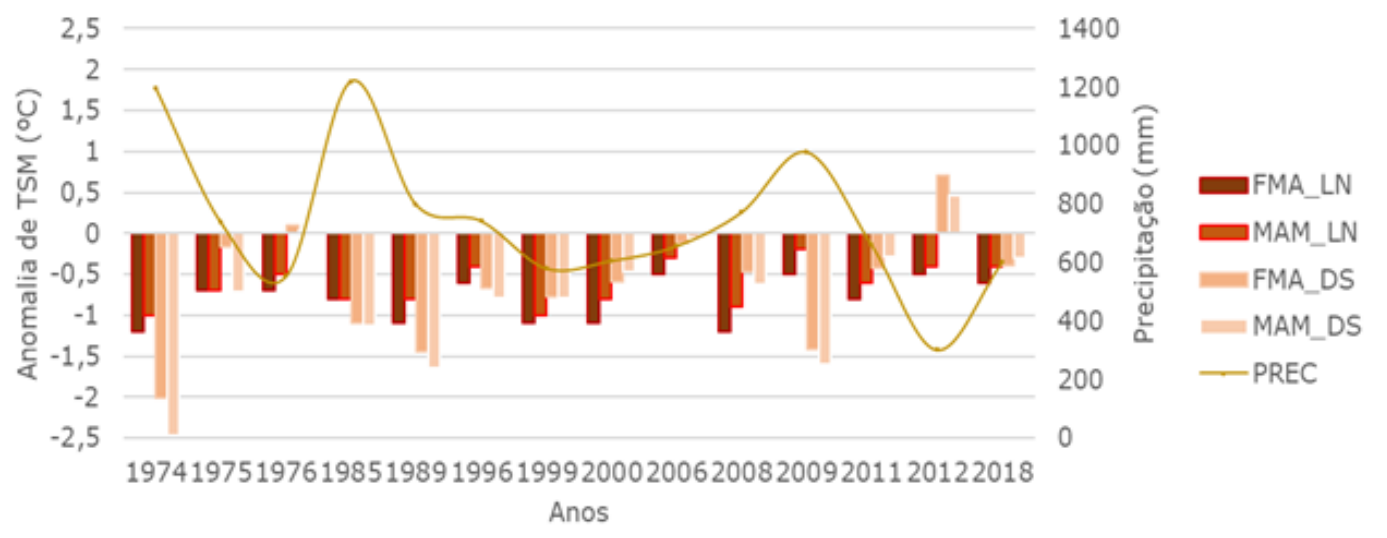

Figura 6 - Índice ONI referente ao LN nos trimestres FMA e MAM (barras vermelhas) e o DS em FMA e MAM (barras laranjas) e a Precipitação média em FMAM em anos de LN (linha) no Ceará. Fonte: FUNCEME/NOAA. 


\section{DESVIOS PERCENTUAIS DE PRECIPITAÇÃO MENSAL EM ANOS DE LN}

A distribuição das chuvas nos meses que compõem a estação chuvosa, FMAM, pode ser analisada através dos valores dos desvios percentuais em anos de LN (Figura 7). Assim como nos anos de EN (Figura 5), ressalta-se que os quatro meses possuem valores médios mensais diferentes e consequentemente, as variações apresentam pesos diferentes em cada mês.

Analisando mensalmente as variações da precipitação em anos de LN (Figura 7), os desvios percentuais correspondentes ao mês de fevereiro, em sua maioria, comportam-se com valores dentro e acima do esperado para o período, com exceção do ano de 1989. Os anos com maiores desvios positivos de chuva foram 1974, 1976, 1985 e 2018. Apenas no ano de 1974, constatou-se a presença de um LN de intensidade moderada no trimestre FMA. Nesse mesmo ano, a anomalia do DS apresentou valores trimestrais entre $-2^{\circ} \mathrm{C}$ e $-2,5^{\circ} \mathrm{C}$, $\mathrm{O}$ que foi determinante para que as chuvas do mês atingissem aproximadamente $50 \%$ da média histórica para o quadrimestre FMAM. O ano de 2018 , diferente dos outros, esteve com o DS neutro com um evento de LN de intensidade fraca. Mesmo com as condições de neutralidade no OA, esse mês foi bastante significativo neste ano, pois contribuiu com 30\% das chuvas em FMAM, visto que março e maio de 2018 tiveram desvios negativos.

O mês de março apresentou valores dentro da média em grande parte dos anos observados (Figura 6). Destacam-se os anos de 1974, 1985 e 2008, com desvios positivos de 50\%. Com exceção de 1985 , o LN se mostrou com intensidade moderada pelo menos no trimestre FMA e DS com anomalias negativas, como visto anteriormente. Em três anos, 2011, 2012 e 2018, as chuvas ficaram abaixo do esperado nesse mês, resultando em desvios negativos que variam entre $20 \%$ e $55 \%$. Esses anos, apresentaram em comum, LN de intensidade fraca ou $L N$ de intensidade fraca a neutro nos trimestres FMA e MAM. No OA, o DS, esteve em 2011 e 2018 neutro e levemente positivo em 2012. É interessante salientar que, tanto em 2012 e 2018, os desvios negativos começam a intensificar em março, persistindo pelos dois meses seguintes, abril e maio, impactando em problemas de falta de água, abastecimento de água e perda da produção, principalmente em 2012, como discutido por Martins e Magalhães (2016).

Abril e maio são os meses em que os desvios oscilam mais dentro da estação chuvosa (Figura 6). Percebe-se que os desvios positivos em abril e maio, em anos de chuvas acima da média, são altos e podem chegar ou ultrapassar os 150\%, como em 1974, 1985 e 2009, já que o DS apresentava valores de anomalia negativas em FMA e MAM e parece se sobrepor a condição do LN, como visto por Lucena et al. (2011). Da Costa et al. (2015), no entanto, destacaram a influência do $L N$ na frequência dos extremos de precipitação nesses dois meses. Por outro lado, os desvios negativos, nos anos de 1976, 2012 e 2018, concentram-se em situações em que DS esteve neutro ou positivo nos dois trimestres. Ressalta-se, ainda, que na maioria dos casos, o mês de maio parece seguir a tendência de abril, ou seja, quando se tem desvios positivos (negativos) de chuvas em abril, também, nota-se uma continuidade no mês seguinte, como exemplo, tem-se os anos de 2006, 2009, 2011, com desvios positivos e 1976, 2012 e 2018 com desvios negativos. 



Figura 7 - Desvio percentual de precipitação mensal no Ceará em anos de LN. Fonte: FUNCEME.

\section{CONSIDERAÇÕES FINAIS}

Entender como as chuvas se comportam durante a estação chuvosa no Ceará e a sua relação com os OP e OA é de grande importância para diversos setores como agricultura e os recursos hídricos. $E$, além disso, essas informações podem auxiliar as previsões climáticas de forma complementar para os anos futuros. Sendo assim, os resultados observados mostraram que nem todos os eventos de EN acarretaram em déficit de chuvas no Ceará. A exemplo disso, destaca-se os anos de 1995 e 2019, nos quais a combinação de EN de intensidade fraca e DS negativo impactaram em chuvas dentro e acima da média.

Ao mesmo tempo, a associação de EN moderado e forte com DS positivo ou neutro foi o principal responsável por chuvas abaixo da normal histórica. Por outro lado, em anos de LN, observou-se a importância do OA a partir do DS, já que nos anos em que o DS esteve com anomalias trimestrais negativas representativas, os volumes médios de precipitação apresentaram desvios percentuais positivos de chuva em torno dos $100 \%$ no quadrimestre FMAM. As chuvas dentro e abaixo da média em anos de LN estão ligadas com a presença de LN de intensidade fraca até o trimestre FMA e neutralidade em MAM e um DS neutro ou positivo.

\section{AGRADECIMENTOS}

Os autores agradecem a Fundação Cearense de Meteorologia e Recursos Hídricos pelo suporte à pesquisa. 


\section{REFERÊNCIAS}

ALVES, J. M. B. Características Intrasazonais da Precipitação no Estado do Ceará em Anos de Contrastes Climatológicos. In: Congresso Brasileiro de Agrometeorologia, XI, Florianópolis, 1999. Anais. Sociedade Brasileira de Agrometeorologia, 1999.

ALVES, J. M. B. Reanálise de índices de temperatura da superfície do mar no Atlântico e Pacífico tropicais potenciais indicadores da qualidade da estação chuvosa do setor norte do Nordeste do Brasil. Revista brasileira de Agrometeorologia, v. 10, n. 1, p. 179-187, 2002.

ALVES, J. M. B; REPELLI, C.A. A variabilidade pluviométrica no setor norte do Nordeste e os eventos El Niño-Oscilação Sul (ENOS). Revista Brasileira de Meteorologia, v. 7, n. 2, p. 583-592, 1992.

ALVES, J. M. B., SOUZA, E. B., REPELLI, C. A., VITORINO, M. I., FERREIRA, N. S. Episódios de La Niña na bacia do Oceano Pacífico Equatorial e a distribuição sazonal e intrasazonal das chuvas no setor norte do Nordeste brasileiro. Revista Brasileira de Meteorologia, v. 12, n. 1, p. 63-76, 1997.

ALVES, J. M. B., CAMPOS, J. N. B., FERREIRA, F. F., STUDART, T. M. C. As chuvas de janeiro/2004 no Nordeste do Brasil, suas características atmosféricas e seus impactos nos recursos hídricos da região. VII Simpósio de Recursos Hídricos do Nordeste, v. 30, p. 1-19, 2004.

ANDREOLI, R. V., KAYANO, M. T., GUEDES, R. L., OYAMA, M. D., ALVES, M. A. S. A influência da temperatura da superfície do mar dos Oceanos Pacífico e Atlântico na variabilidade de precipitação em Fortaleza. Revista Brasileira de Meteorologia, v. 19, n. 3, p. 337-344, 2004.

DA SILVA COSTA, M., LIMA, K. C., DE MENDONÇA ANDRADE, M., ANDRADE, W. Tendências observadas em extremos de precipitação sobre a região Semiárida do Nordeste do Brasil. Revista Brasileira de Geografia Física, v. 8, n. 05, p. 1321-1334, 2015.

FERREIRA, A. G., DA SILVA MELLO, N. G. Principais sistemas atmosféricos atuantes sobre a região Nordeste do Brasil e a influência dos oceanos Pacífico e Atlântico no clima da região. Revista Brasileira de Climatologia, v. 1, n. 1, 2005.

FUNCEME, Fundação Cearense de Meteorologia e Recursos Hídricos. Disponível em: <www.funceme.br>. Acesso em: setembro de 2020.

LUCENA, D. B, GOMES FILHO, M. F., SERVAIN, J. Avaliação do impacto de eventos climáticos extremos nos Oceanos Pacífico e Atlântico sobre a estação chuvosa no nordeste do Brasil. Rev. bras. meteorologia, p. 297-312, 2011.

MARENGO, J. A. Vulnerabilidade, impactos e adaptação à mudança do clima no semi-árido do Brasil. Parcerias estratégicas, v. 13, n. 27, p. 149-176, 2010.

MARENGO, J. A., ALVES, L. M., BESERRA, E. A., LACERDA, F. F. Variabilidade e mudanças climáticas no semiárido brasileiro. Recursos hídricos em regiões áridas e semiáridas, v. 1, 2011.

MARENGO, J. A., CUNHA, A.P., ALVES, LINCOLN M. A seca de 2012-15 no semiárido do Nordeste do Brasil no contexto histórico. Climanálise, v. 3, n. 1, p. $1-6,2016$. 
MARTINS, E. S. P. R; MAGALHÃES, A. R. A seca de 2012-2015 no Nordeste e seus impactos. Parcerias Estratégicas, v. 20, n. 41, p. 107-128, 2016.

MARTINS, E. S. P. R; VASCONCELOS, F. C. O clima da região Nordeste entre 2009 e 2017: monitoramento e previsão. Parcerias estratégicas, v. 22, n.44, p.63-80, 2017.

MARTINS, E. S. P. R; MAGALHÃES, A. R; FONTENELE, D. A seca plurianual de 2010-2017 no Nordeste e seus impactos. Parcerias Estratégicas, v. 22, n. 44, p. 17-40, 2017.

MEDEIROS, F. J. D. Aspectos dinâmicos da atmosfera associados a seca de 2012-2016 no Nordeste do Brasil. 2019. Dissertação de Mestrado. Brasil.

MOURA, A. D., SHUKLA, J. On the dynamics of droughts in northeast Brazil: Observations, theory and numerical experiments with a general circulation model. Journal of Atmospheric Sciences, v. 38, n. 12, p. 2653-2675, 1981.

NOBRE, P., SHUKLA, J. Variations of sea surface temperature, wind stress, and rainfall over the tropical Atlantic and South America. Journal of climate, v. 9, n. 10, p. 2464-2479, 1996.

REBOITA, M. S; SANTOS, I. Influência de alguns padrões de teleconexão na precipitação no norte e nordeste do Brasil. Revista Brasileira de Climatologia, v. 15, 2015.

RODRIGUES, R. R., HAARSMA, R. J., CAMPOS, E. J., AMBRIZZI, T. The impacts of inter-El Niño variability on the tropical Atlantic and northeast Brazil climate. Journal of Climate, v. 24, n. 13, p. 3402-3422, 2011.

SERVAIN, J. Simple climatic indices for the tropical Atlantic Ocean and some applications. Journal of Geophysical Research: Oceans, v. 96, n. C8, p. 1513715146, 1991 